\title{
Application of Abrupt Change Detection in Power Systems Disturbance Analysis and Relay Performance Monitoring
}

\author{
Abhisek Ukil, Student Member, IEEE, and Rastko Živanović, Member, IEEE
}

\begin{abstract}
This paper describes the application of the abrupt change detection technologies to detect the abrupt changes in the signals recorded during disturbances in the electrical power network of South Africa for disturbance analysis and relay performance monitoring. The aim is to estimate the time instants of the changes in the signal model parameters during the prefault condition, after initiation of fault, after the circuit-breaker opening and autoreclosure, etc. After these event-specific segmentations, the synchronization of the different digital fault recorder recordings are done based on the fault inception timings. The synchronized signals are segmented again. This synchronized segmentation is the first step toward automatic disturbance recognition, facilitating further complex feature vector analysis and pattern recognition. Besides, the synchronized, segmented recordings can be directly used to analyze certain kinds of disturbances and monitor the relay performance. This paper presents many practical examples from the power network in South Africa.
\end{abstract}

Index Terms-Abrupt change detection, disturbance analysis, relay performance, wavelet transform (WT).

\section{INTRODUCTION}

A UTOMATIC disturbance recognition and analysis from the recordings of digital fault recorders (DFRs) play a significant role in fast fault clearance, helping to achieve a secure and reliable electrical power supply. Segmentation of the fault recordings by detecting the abrupt changes in the characteristics of the fault recordings, obtained from the DFRs of the power network in South Africa, is the first step toward automatic disturbance recognition and analysis. Besides facilitating the disturbance analysis, abrupt change detection-based segmentation also helps in monitoring the performances of the protective relays during the disturbances.

To accomplish the abrupt change detection, we propose the use of the wavelet transform to transform the original fault signal into finer wavelet scales, followed by a progressive search for the largest wavelet coefficients on that scale [1]. Large wavelet coefficients that are co-located in time across different scales provide estimates of the changes in the signal parameter. The change time instants can be estimated by the number of wavelet coefficients that exceed a given threshold

Manuscript received May 25, 2005; revised February 2, 2006. This work was supported by the National Research Foundation (NRF), South Africa. Paper no. TPWRD-00312-2005.

The authors are with the Tshwane University of Technology, Pretoria 0001, South Africa (e-mail: abhiukil@yahoo.com; zivanovr@yahoo.com).

Digital Object Identifier 10.1109/TPWRD.2006.887096 (which is equal to the first-order approximation of the "universal threshold" of Donoho and Johnstone [2]).

After the segmentation, the segmented fault recordings, from the different DFRs triggering for the same disturbance are synchronized depending on the fault inception timings. This is essential as the time bases of the DFRs triggering for the same disturbance are not perfectly synchronized [3]. The synchronized analog signals are segmented once again using the abrupt change detection technologies. The synchronized, segmented analog signals along with the respective recorded binaries, also synchronized following the corresponding analog signals, can be used for performing certain disturbance analysis and monitoring the relay performance during the disturbance.

The remainder of this paper is organized as follows. In Section II, automatic disturbance analysis as an application domain is discussed. In Section III, abrupt change detection using the wavelet transform and threshold method is reviewed. Section IV presents the synchronization technique for the different DFR recordings for the same event, already segmented as described in Section III. Monitoring of the relay performance is presented with examples in Section V. The analysis of certain kinds of disturbances directly from the synchronized recordings are discussed with examples in Section VI. Discussion on results and future directions are presented in Section VII, and conclusions are given in Section VIII.

\section{Automatic Disturbance Analysis}

Automatic analysis of the disturbances in the Eskom (South African power utility) transmission network of South Africa depends on the DFR recordings. Presently, 98\% of the Eskom transmission lines are equipped with the DFRs on the feeder bays, with an additional few installed on the static var compensators (SVCs) and $95 \%$ of these are remotely accessible via a X.25 communication system [4]. The DFRs trigger due to reasons such as power network fault conditions, protection operations, breaker operation, and the like. Each DFR recording typically consists of 32 points binary information and analog information in the form of voltages and currents per phase as well as the neutral current. Following IEEE COMTRADE standard [5], the DFR recordings are provided as input to the fault analysis software which uses discrete Fourier analysis and superimposed current quantities [4].

The purpose of this study is to augment the existing semiautomatic fault analysis system with more robust and accurate algorithms and techniques to make it fully automated. So we 
would first apply the abrupt change detection algorithms to segment the fault recordings into different event-specific segments, namely, prefault segment, after the initiation of fault, after circuit-breaker (CB) opening, after autoreclosure of the CBs. Then, we would construct the appropriate feature vectors for the different segments; finally, the pattern-matching algorithm would be applied using those feature vectors to accomplish the fault recognition and disturbance analysis tasks.

The first step toward the proposed automatic fault analysis, namely the abrupt change detection-based segmentation, is quite critical for improving the fault recognition rate, automatic analysis quality, and the speed of operation. It also provides a great scope for monitoring the performances of the protective relays and analyzing certain kinds of disturbances directly from the segmented recordings before conforming to any further significant and complex feature vector analysis. These are focussed in the scope of this paper.

\section{ABrupt ChANGE DeteCtion}

The authors have already discussed different technologies for abrupt change detection in a comparative manner in [6]. The techniques are broadly categorized as simple methods, linear model-based approach, model-free approach, and nonparametric approach [6]. Among these techniques, the nonparametric approach, namely, the wavelet transform (WT), appears to be the most suitable for this specific application.

A detailed description of the abrupt change detection using the WT and threshold checking can be referred to in [7]. We only provide the summary of the abrupt change detection using the WT in the following sections.

\section{A. Wavelet Decomposition}

The continuous wavelet transform (CWT) is defined as the sum over all time of the signal multiplied by scaled and shifted versions of the wavelet function $\psi$. The CWT of a signal $x(t)$ is defined as

$$
\operatorname{CWT}(a, b)=\int_{-\infty}^{\infty} x(t) \psi_{a, b}^{*}(t) d t
$$

where

$$
\psi_{a, b}(t)=|a|^{-1 / 2} \psi((t-b) / a) .
$$

$\psi(t)$ is the mother wavelet, the asterisk in (1) denotes a complex conjugate, and $a, b \in R, a \neq 0,(R$ is the real continuous number system) are the scaling and shifting parameters, respectively. The discrete wavelet transform (DWT) is given by choosing $a=a_{0}^{m}, b=n a_{0}^{m} b_{0}, t=k T$ in (1) and (2), where $T=1.0$ and $k, m, n \in Z,(Z$ is the set of positive integers)

$$
\operatorname{DWT}(m, n)=a_{0}^{-m / 2}\left(\sum x[k] \psi^{*}\left[\left(k-n a_{0}^{m} b_{0}\right) / a_{0}^{m}\right]\right) .
$$

We apply the multiresolution signal decomposition (MSD) [8] technique and the quadrature mirror filter (QMF) [8] banks to decompose the fault signals from the DFRs into localized and detailed representations in the form of wavelet coefficients. Daubechies 1 and 4 [9] wavelets are used as the mother wavelets. Among many other choices of the mother wavelets (e.g., Coiflets, Meyer wavelet, Gaussian wavelet, Mexican hat wavelet, Morlet wavelet, etc. [9]), Daubechies 1 and 4 wavelets have been chosen because Daubechies wavelets are compactly supported wavelets with extremal phase and the highest number of vanishing moments for a given support width; also, the associated scaling filters are minimum-phase filters [7]. So, from the point of view of fast implementation and varying patterns of the fault signals, Daubechies wavelets appear to be the optimal choice for the mother wavelet for this specific application [7].

After transforming the original fault signal using the mother wavelets and DWT, we obtain the smoothed and detailed versions. The detailed version, called the WT coefficient, is used for threshold checking to estimate the change time instants [7].

\section{B. Application of Threshold Method}

As wavelet coefficients are the changes of the averages, so a coefficient of large magnitude implies a large change in the original signal [7]. The change time instants can be estimated by the instants when the wavelet coefficients exceed a given threshold which is equal to the first-order approximation of the "universal threshold" of Donoho and Johnstone [2]. The universal threshold $T$ is given by

$$
T=\sigma \sqrt{2 \log _{e} n},
$$

where $\sigma$ is the median absolute deviation of the wavelet coefficients [2], and $n$ is the number of samples of the wavelet coefficients. The universal threshold is an adaptive technique and the threshold depends on the wavelet coefficients and not the whole signal; hence, the frequency content of the fault signal and the wavelet filter would not affect the point the signal reaches the universal threshold.

\section{Smoothing Filtering}

After threshold checking, to indicate the change time instants as unit impulses, the following smoothing filtering operations are performed sequentially [7].

- It removes confusing multiple close spikes and combines them into single unit impulse.

- It removes any unwanted glitches which can otherwise result in false positives for the abrupt changes. Here, false positives refer to wrong spikes which indicate possible segments when there should not be any.

- Based on the modeling of the segments, analysis is performed for estimating the event-critical change instants, rejecting others.

\section{Application Example}

Fig. 1 shows the abrupt change detection result using the WT and threshold method. In Fig. 1, the original DFR recording from the Eskom transmission network for the voltage during a phase-to-ground fault in the BLUE-phase, sampled at a frequency of $2.5 \mathrm{kHz}$ [4], is shown in plot (i); wavelet coefficients for this fault signal and the universal threshold (dashed) are shown in plot (ii) and the change time-instants as unit impulses computed using the threshold checking (plot ii) followed by smoothing filtering [7] are shown in plot (iii). The time instants of the changes in the signal characteristics in plot (iii) indicate the different signal segments owing to different events 


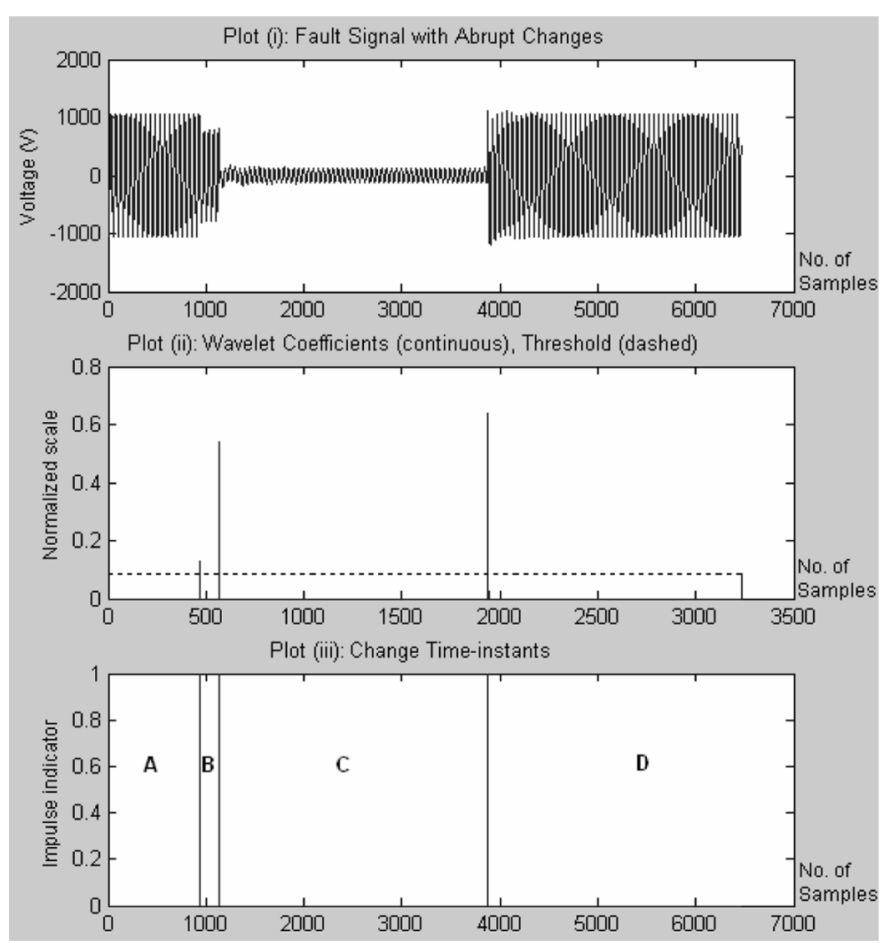

Fig. 1. Abrupt change detection-based segmentation of the BLUE-phase voltage recording during a phase-to-ground fault.

during the fault (e.g., segment $\mathrm{A}$ indicates the prefault section and the fault inception, segment $B$ is the fault, segment $C$ is the opening of the $\mathrm{CB}$, and segment $\mathrm{D}$ is the autoreclosing of the $\mathrm{CB}$ and system restore). Ninety-eight percent segmentation accuracy has been achieved using the wavelet method, with the average computation time being $0.431 \mathrm{~s}$ using MATLAB $^{\circledR}$ [7].

\section{SYNCHRONIZATION}

Usually many DFRs employed for different substations trigger during any abnormal condition in the power network. All of these simultaneous recordings, typically a 3-s duration covering the disturbance event [4], differ by some time delays. First, all of these simultaneous recordings are segmented using the abrupt change detection method described before. But before any further global analysis, an important step is required, namely "Synchronization." Eighty percent of the DFRs in the Eskom power network are synchronized with the global positioning system (GPS) [4] to compensate for the constant time drift within the monitor. However, the synchronization step is essential as the time bases of the DFRs triggering the same disturbance are not perfectly synchronized with each other [3]. This can lead to erroneous conclusions especially when analyzing the performances of the protective relays, etc.

In Fig. 2, we show the voltage recordings of three different DFRs triggering for the same phase-to-ground fault involving the BLUE phase.

First, all recordings are segmented using the abrupt change detection method as described in Section III. And this is reflected in all the three plots in Fig. 2 by the dashed vertical lines, segmenting the voltage signals into segments such as A, B, C, and D. For all of them, segment $A$ indicates the prefault section and the fault inception, segment $B$ the fault, segment $C$ opening

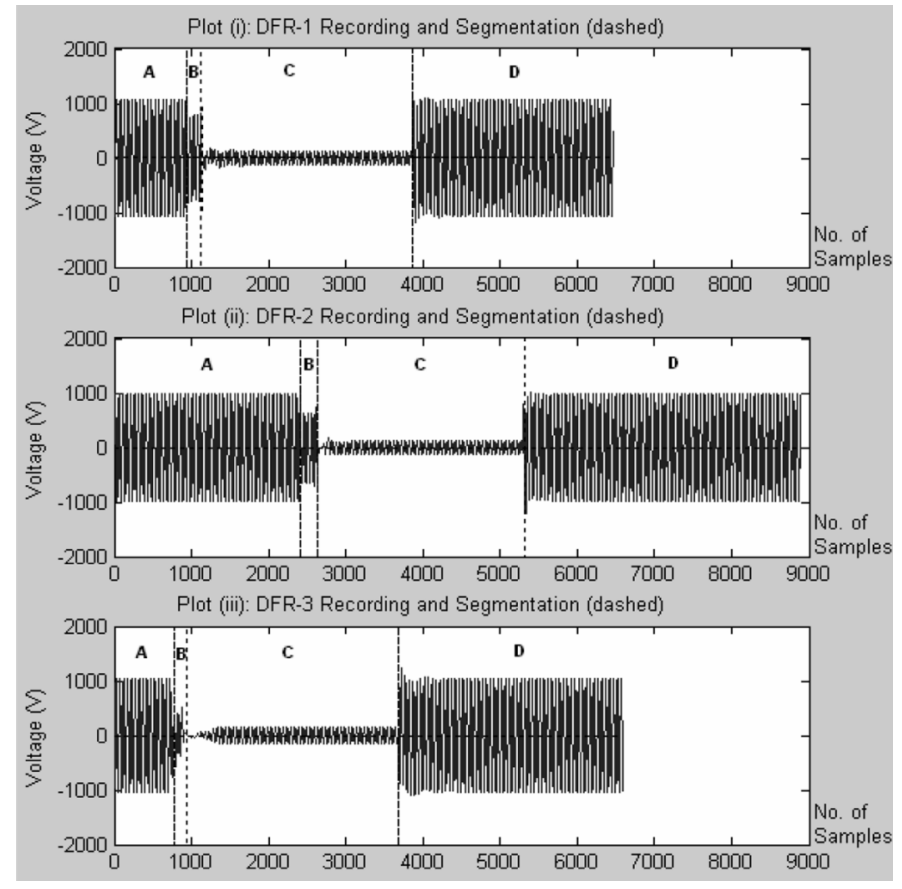

Fig. 2. Three segmented but unsynchronized DFR voltage recordings for the same phase-to-ground fault.

TABLE I

FAULT INCEPTION TIME OF THE DFR RECORDINGS SHOWN IN FIG. 2

\begin{tabular}{ccc}
\hline Recordings & $\begin{array}{c}\text { Fault Inception } \\
\text { Time } \\
\text { (Sample No.) }\end{array}$ & $\begin{array}{c}\text { Fault Inception } \\
\text { Time } \\
\text { (ms) }\end{array}$ \\
\hline DFR-1 & 938 & 375.2 \\
DFR-2 & 2410 & 964 \\
DFR-3 & 770 & 308 \\
\hline
\end{tabular}

of the $\mathrm{CB}$, and segment $\mathrm{D}$ autoreclosing of the $\mathrm{CB}$ and system restore. Clearly from Fig. 2, all of these segments are not synchronized for the three recordings although they essentially represent the same event.

To synchronize the recordings for further analysis, we will use fault inception timing (i.e., the borderline between segment $\mathrm{A}$ and $\mathrm{B}$ in all of the segmented recordings). It is to be noted that in Fig. 2, the $X$-axis indicates the number of samples, so we have to divide it by the sampling frequency of $2.5 \mathrm{kHz}$ [4] to get the fault inception time in milliseconds. Table I lists the fault inception timings of the three DFR recordings shown in Fig. 2.

The complete synchronization algorithm, based on the fastest fault inception time, is described below.

- First, we segment the different DFR recordings for the same disturbance using abrupt change detection.

- Then, we estimate the individual fault inception timings of the segmented but unsynchronized recordings.

- We choose the recording with the minimum fault inception time as the reference one. (In this case, it is the DFR-3 recording as evident from Table I).

- We synchronize the rest of the recordings with the reference recording by equating their fault inception times with the reference fault inception time (i.e., we left-shift the rest 


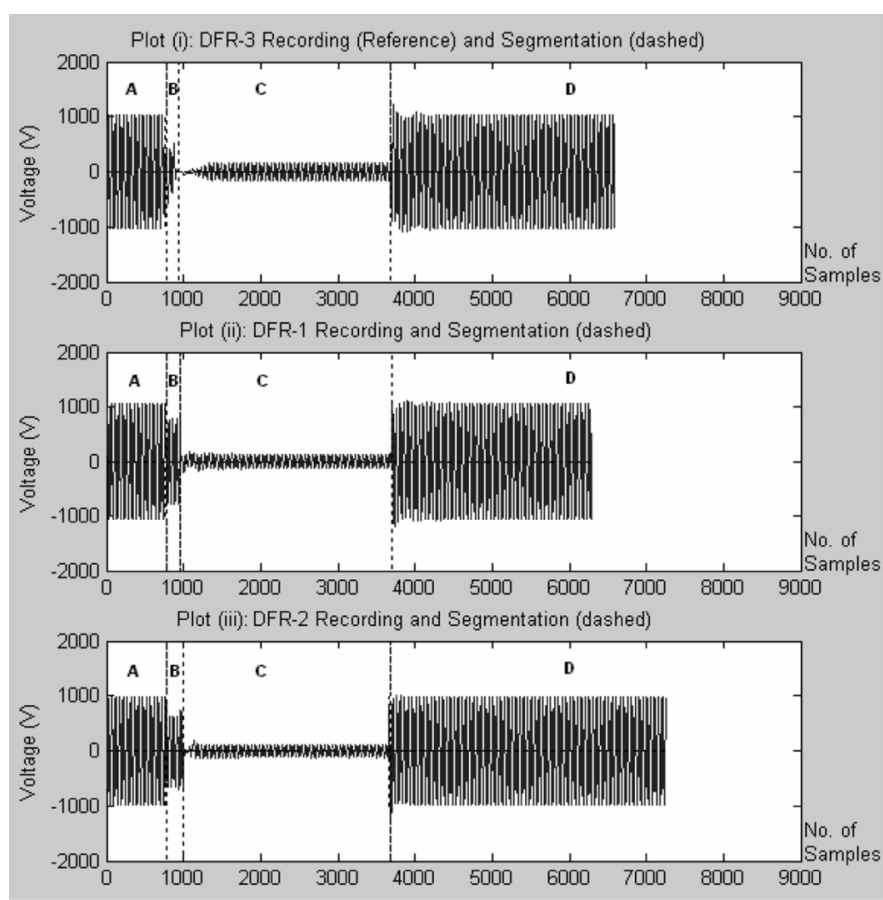

Fig. 3. Three segmented and synchronized DFR voltage recordings for the same phase-to-ground fault.

of the recordings, their fault inception times equating to the reference one.

- Then we again perform the abrupt change detection-based segmentation on these synchronized recordings to have the synchronized, segmented recordings for further analysis.

The application of the synchronization algorithm on the unsynchronized recordings shown in Fig. 2 results in the synchronized, segmented recordings as depicted in Fig. 3. The DFR-3 recording with the minimum fault inception time is chosen as the reference (plot i) and the other two recordings are synchronized accordingly.

After synchronizing the analog signals (voltage signals in this case) of the different DFRs, the respective binaries are also synchronized and matched against the synchronized, segmented analog signals. One such example for the DFR-3 recording, analog voltage, and binaries for the fault duration and $\mathrm{CB}$ autoreclosure are shown in Fig. 4.

After this, using the matched binary plots as a cross-check against any possible discrepancies, we estimate the change time instants of the synchronized, segmented analog recordings of the different DFRs. Table II lists the change time instants of the recordings shown in Fig. 3.

\section{Relay Performance Monitoring}

The synchronized, segmented analog signals with the matched synchronized binaries can be used to monitor different performance characteristics of the protective relays (e.g., fastest relay operating time, autoreclosing of the $\mathrm{CBs}$ ).

\section{A. Fastest Relay Operating Time}

To determine the fastest relay operating time, first we need to determine the fault duration. This can be done by estimating the duration of the segment B (fault) in the synchronized analog

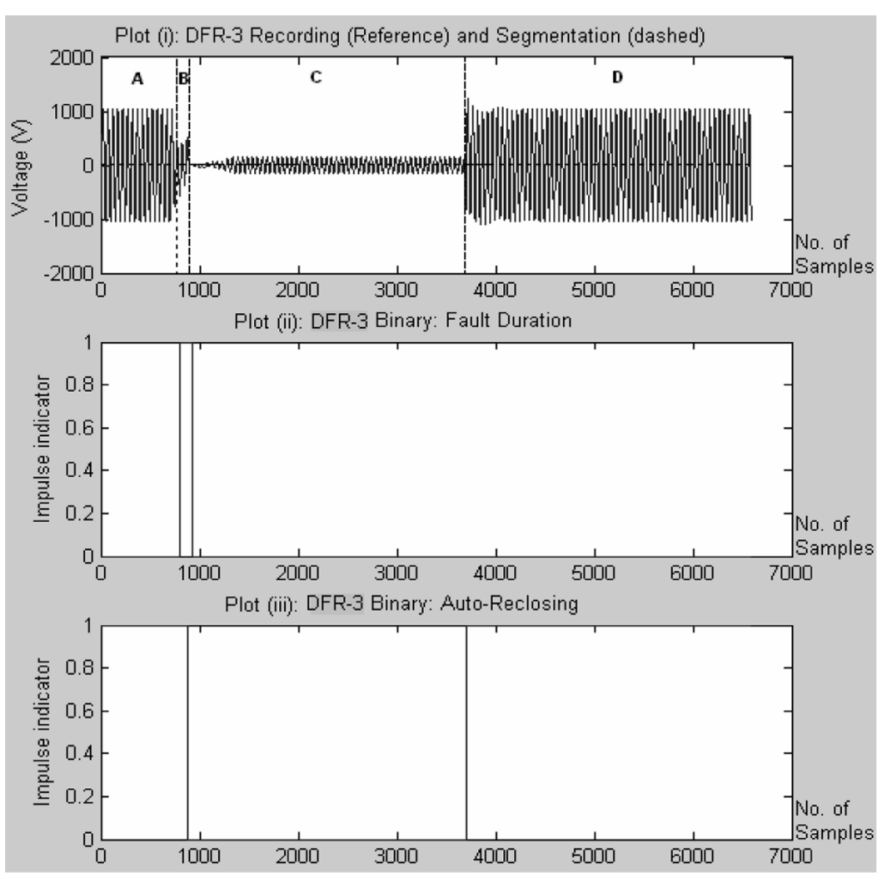

Fig. 4. Synchronized analog voltage recording plot and binary plots for the fault duration and $\mathrm{CB}$ autoreclosing.

TABLE II

Change Time Instants of the Synchronized, SEgmented ANALOG DFR RECORDINGS SHOWN IN FIG. 3

\begin{tabular}{cccc}
\hline Recordings & $\begin{array}{c}\text { Segment A-B } \\
\text { Time-instant } \\
\text { (Sample No.) }\end{array}$ & $\begin{array}{c}\text { Segment B-C } \\
\text { Time-instant } \\
\text { (Sample No.) }\end{array}$ & $\begin{array}{c}\text { Segment C-D } \\
\text { Time-instant } \\
\text { (Sample No.) }\end{array}$ \\
\hline DFR-3 & 770 & 946 & 3688 \\
DFR-1 & 770 & 962 & 3704 \\
DFR-2 & 770 & 992 & 3672 \\
\hline
\end{tabular}

TABLE III

FAUlt DURATION IN THE SYNCHRONIZED, SEgMENTED ANALOG DFR RECORDINGS SHOWN IN FIG. 3

\begin{tabular}{ccc}
\hline Recordings & $\begin{array}{c}\text { Fault Duration } \\
\text { (No. of Samples) }\end{array}$ & $\begin{array}{c}\text { Fault Duration } \\
\text { (ms) }\end{array}$ \\
\hline DFR-3 & $946-770=176$ & 70.4 \\
DFR-1 & $962-770=192$ & 76.8 \\
DFR-2 & $992-770=222$ & 88.8 \\
\hline
\end{tabular}

plots shown in Fig. 3, with the help of Table II. Table III lists the fault duration times of the three DFR recordings shown in Fig. 3 in terms of the number of samples and milliseconds.

For determining the fastest relay operation, we need to select the minimum fault duration from the different synchronized DFR recordings. In this case, this is the DFR-3 fault duration (70.4 ms) from Table III. The formula for calculating the fastest relay operating time is given as follows:

Fastest Relay Operating Time $=$ Fault Duration-Trip Time. (5)

Most of the CBs in the Eskom transmission system are twocycle breakers [4] (i.e., the expected tripping time (theoretically speaking) is in the region of $40 \mathrm{~ms}$ ( $50-\mathrm{Hz}$ system). Although the clearing time depends on parameters such as the fault inception 
TABLE IV

CB AutoReClosing LENGTH OF THE SYNCHRONIZED AND SEGMENTED ANALOG DFR RECORDINGS SHOWN IN FIG. 3

\begin{tabular}{ccc}
\hline Recordings & $\begin{array}{c}\text { Auto-Reclosing } \\
\text { Length } \\
\text { (No. of Samples) }\end{array}$ & $\begin{array}{c}\text { Auto-Reclosing } \\
\text { Length } \\
\text { (ms) }\end{array}$ \\
\hline DFR-3 & $3688-946=2742$ & 1096.8 \\
DFR-1 & $3704-962=2742$ & 1096.8 \\
DFR-2 & $3672-992=2680$ & 1072 \\
\hline
\end{tabular}

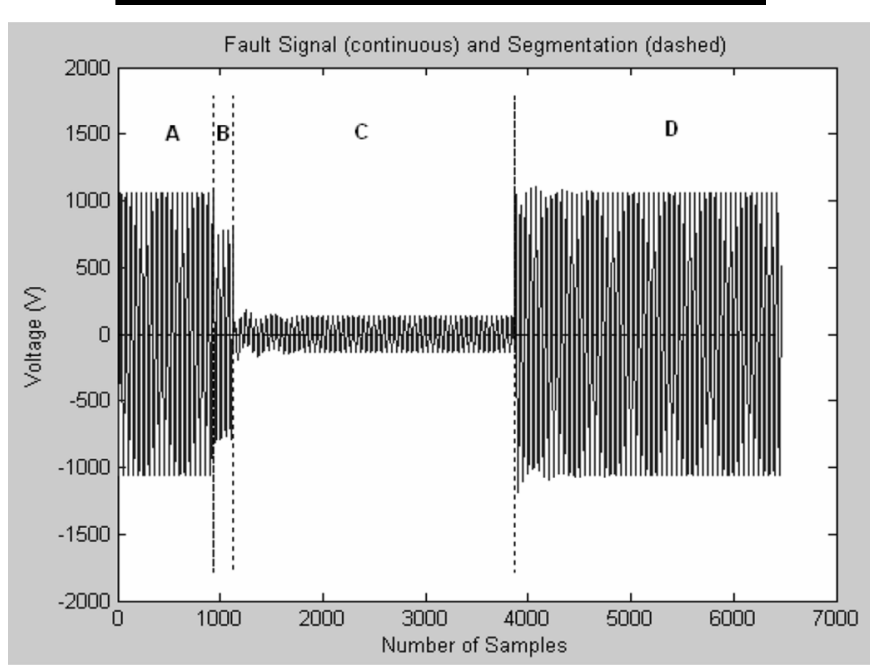

Fig. 5. Segmented analog voltage recording during a phase-to-ground fault cleared successfully.

angle and the current magnitude etc., but using this information, we can compute a theoretical estimate for the fastest relay operating time during the disturbance which, for our example case, is $70.4-40=30.4 \mathrm{~ms}$. Although the theoretical fastest relay operating time calculated this way could be optimistic, it can provide quick insight into the fastest relay operating time from the segmentation step itself as all of the relay operating times are calculated by using the same synchronized segmentation basis.

\section{B. Autoreclosing CBs}

From the synchronized, segmented analog signals and their matched binaries, it is possible to analyze autoreclosing of the CBs.

By comparing the signal parameter values of the segment A and D in the synchronized analog plots as shown in Fig. 3, it can be determined whether the autoreclosing is successful or not following the relay operation. In this case, matching the segment $\mathrm{A}$ and D signal parameters values of the synchronized analog plots in Fig. 3 yields that autoreclosing of the CBs has been successful.

Length of the autoreclosing can be determined by estimating the duration of the segment $\mathrm{C}$ in the synchronized analog plots shown in Fig. 3. Using the synchronized analog plots of Fig. 3 and Table II values, we can compute the length of autoreclosing (segment C) for all three DFR recordings, as listed in Table IV.

\section{Disturbance ANALYSIS EXAMPLES}

Abrupt change detection-based segmentation is the first step toward the automatic fault recognition and disturbance analysis, followed by feature vector construction and pattern matching.

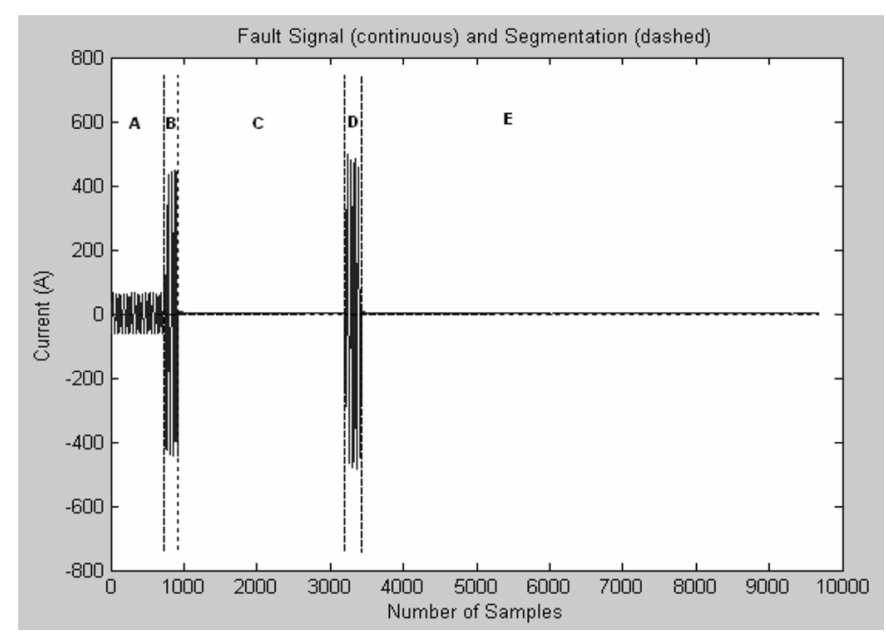

Fig. 6. Segmented analog current recording during a phase-to-ground fault not cleared successfully.

In this section, we would discuss the analysis of certain kinds of disturbances directly from the segmented recordings before conforming to any further significant and complex feature vector analysis.

\section{A. Cleared Single-Phase Fault}

Fig. 5 shows the original DFR recording from the Eskom transmission network for the voltage during a phase-to-ground fault in the RED phase. Segmentations of the voltage signal are indicated by the vertical dashed lines in Fig. 5 .

In Fig. 5, segment A indicates the prefault section and the fault inception, and segment $\mathrm{D}$ indicates autoreclosing of the $\mathrm{CB}$ and system restore. A comparison of the signal parameter values of segment $\mathrm{A}$ and $\mathrm{D}$ shows that the fault was cleared successfully.

\section{B. Uncleared Single-Phase Fault}

By evaluating the induced voltage on the open phase, it can be seen whether the secondary arc is extinguished or not. To sustain the secondary arc, very little voltage is required. Should the breaker close before the secondary arc is extinguished, the fault will reappear and a three-phase trip will be issued [10]. Fig. 6 shows an application example of how the uncleared fault can be evaluated using the abrupt change detection-based segmentation.

In Fig. 6, the current during the phase-to-ground fault in the BLUE phase is shown along with the segmentations. The reappearance of the uncleared fault is evident from the comparison of the segment B and D, representing the fault and segment C, representing the breaker opening and reclosing.

\section{CB Restrike}

Restriking of a CB normally occurs when the breaker has to interrupt capacitive current. A line breaker will interrupt capacitive current when it is the second breaker to open, meaning that the remote end breaker is already open. During this condition, only charging current will flow, which is capacitive. During the switching of capacitive current, a very high voltage can be seen across the breaker contacts which result in a reappearance of the arc. The reappearance is called a restrike if it occurs $5 \mathrm{~ms}(1 / 4$ 


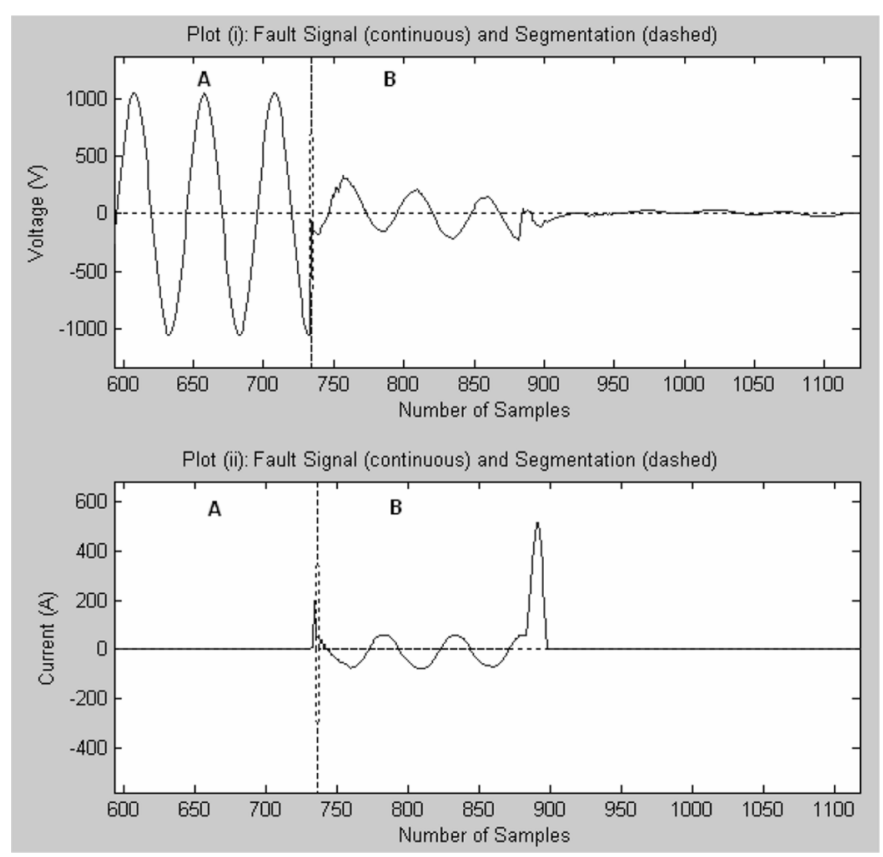

Fig. 7. Voltage (plot i) and current (plot ii) recordings during a CB restrike.

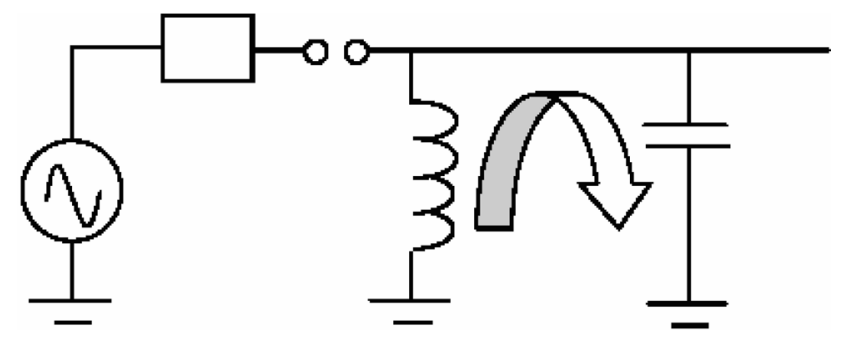

Fig. 8. Schematic oscillating circuit diagram for the reactor ring down phenomenon.

of a cycle for the $50-\mathrm{Hz}$ system) after breaker contact separation. The end that is restriking can be determined by examining the current waves. The voltage will cross through zero instantaneously and this will be associated with a spike in the current [10].

In Fig. 7, we show the voltage and current recordings during a CB restrike in plots (i) and (ii), respectively. Using the abrupt change detection, both the voltage and current recordings are segmented into two segments, A and B (i.e., before and after the restrike of the $\mathrm{CB}$ ). It is to be noted that the segments of the voltage and the current are matched. A closer look at the segment B of the segmented current plot also shows the spike, indicating the restriking end.

\section{Reactor Ring Down}

When a line reactor is connected to a line and the CBs are opened at both ends, the voltage does not disappear; instead, an oscillating voltage waveform can be found which slowly reduces in magnitude. This phenomenon is called reactor ring down [10]. It is a result of the interaction between the reactor and the capacitance of the line. This forms an oscillatory circuit. A schematic diagram is shown in Fig. 8.

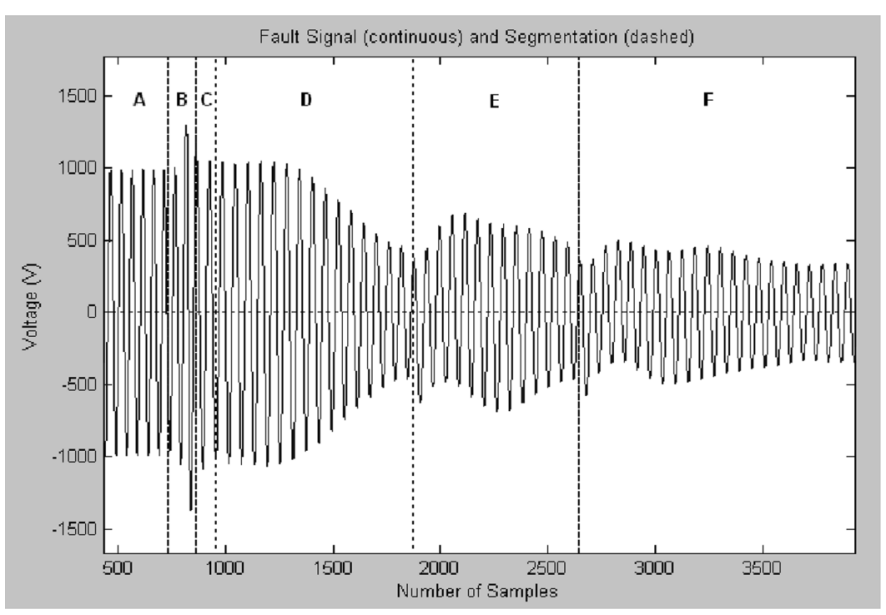

Fig. 9. Voltage recording during a reactor ring down. The waveform is oscillating in nature and slowly reduces in magnitude.

Fig. 9 shows the segmented voltage recording during a rector ring down incident. The segmentations are shown as vertical dashed lines.

Usually there are many segments for the oscillating signals recorded during the reactor ring down phenomenon and this is evident from Fig. 9. Comparisons of the signal parameter values for the segments B, C, D, E, and F in Fig. 9 indicate that the signal is oscillating and slowly reduces in magnitude.

\section{E. Capacitive Voltage Transformer Transient Behavior}

Capacitive voltage transformer (CVT) transient behavior is due to the discharge of energy stored in the capacitive and inductive elements of the CVT when there is a sudden change in the primary voltage. The transient behavior is seen as oscillations in the secondary voltage. The transients are influenced by the burden on the CVT. For a resistive burden, the transients are normally very small and die down very quickly. For zero or small burdens, the transients are very prominent [10].

Fig. 10 shows a segmented voltage recording for the transient behavior of the CVT. From Fig. 10, it is to be noted that the transient behavior is reflected in the segment B. For zero or small burdens, the transients are very prominent as can be seen at the start of the segment B and start of segment $\mathrm{C}$.

\section{F. Energizing a Transformer}

Energizing a transformer often goes together with high magnetizing inrush currents. Transformer protection must be set so that the transformer does not trip for this inrush current. This high current is due to the remnant flux in the transformer core when it was switched out and it depends where on the sine wave the transformer is switched back in [10].

The segmented current recording for energizing a transformer is shown in Fig. 11. In Fig. 11, the current signal is segmented into two segments, namely segment $\mathrm{A}$ and $\mathrm{B}$ (i.e., energizing the transformer before and after). A closer look at segment B yields the fact.

\section{G. Analysis of Power Signals From Mexican Network}

Ruiz-Vega et al. discussed the use of nonlinear, nonstationary analysis techniques to characterize forced interarea 


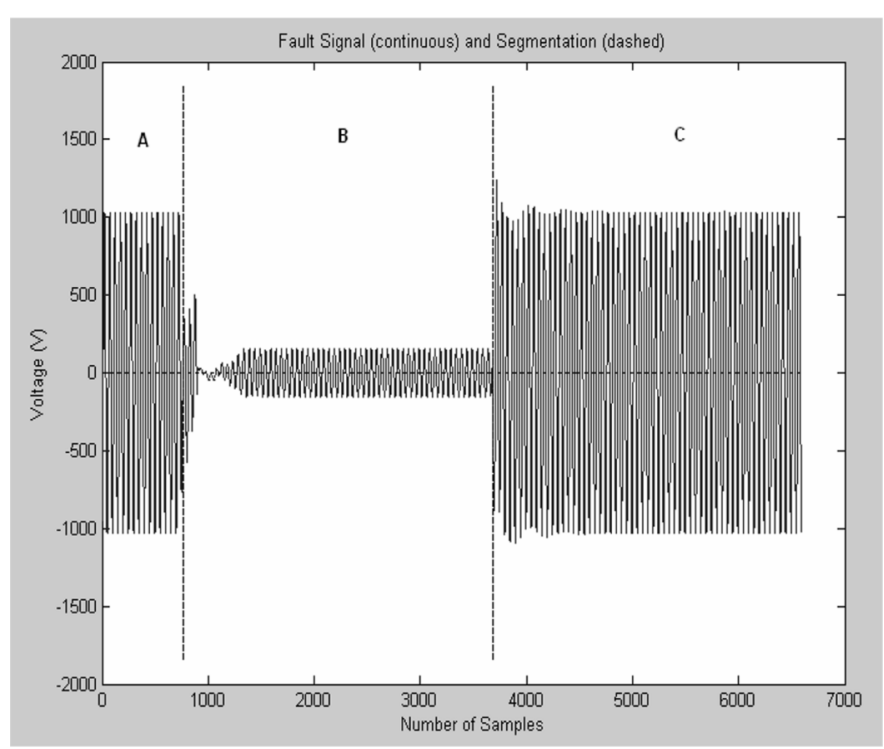

Fig. 10. Segmented voltage recording for the transient behavior of the capacitive voltage transformer (CVT).

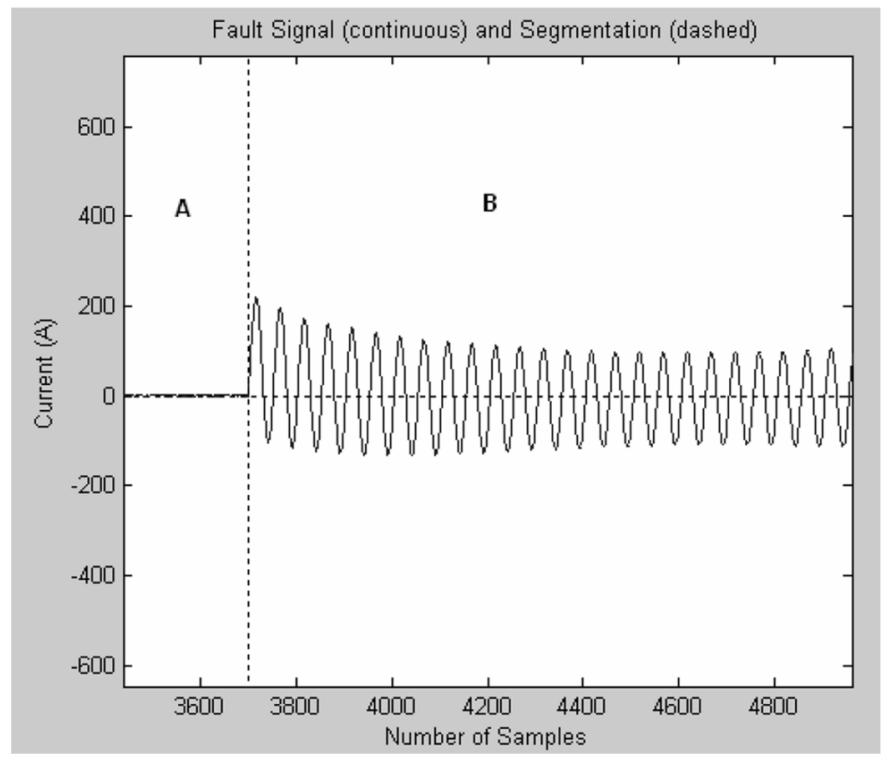

Fig. 11. Segmented current recording reflecting the energization of a transformer, which is associated with high magnetizing inrush currents.

oscillations problem in power systems, recorded in the Mexican interconnected system [11]. In collaboration with them, we also tested our segmentation algorithms on the power oscillation signals obtained from the Mexican interconnected system (MZD-DGD) [11] as discussed in detail in [12]. Fig. 12 shows the segmentation result using the wavelet method.

\section{Discussions AND FUTURE DiRECTIONS}

In this paper, we have discussed the different applications of the abrupt change detection-based automatic signal segmentation techniques in the power systems domain. In doing so, the following assumptions have been made for all of the techniques and application examples in this paper.

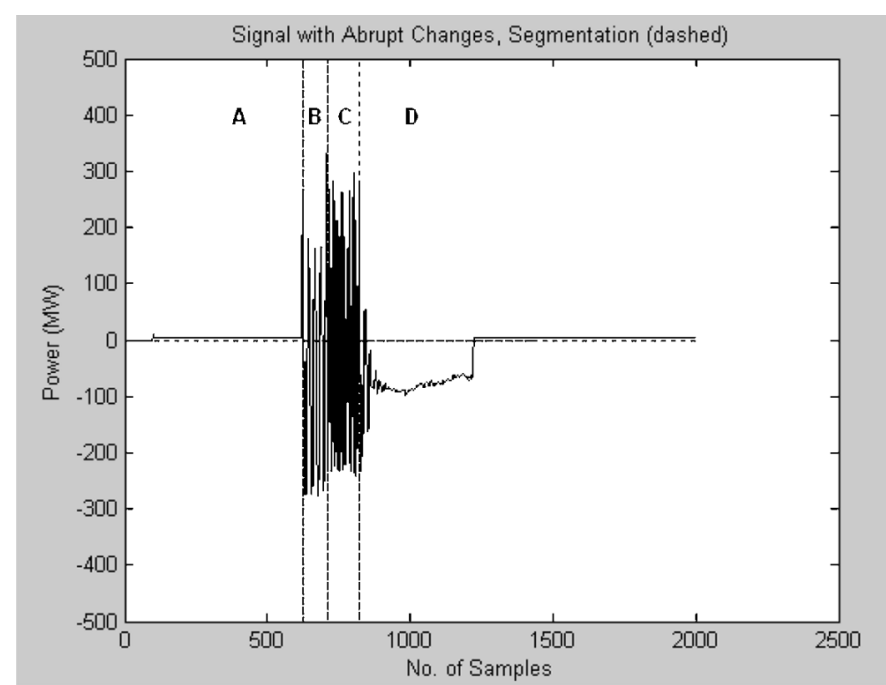

Fig. 12. Segmentation of the power oscillation signal from the Mexican power network, using the wavelet method.

1) The application domain is the electrical power network in South Africa for the purpose of automatic disturbance recognition and analysis.

2) The signals are from a $50-\mathrm{Hz}$ frequency power system.

3) All of the disturbance recordings from the DFRs are sampled at a $2.5-\mathrm{kHz}$ sampling frequency.

4) The DFRs typically record for a duration of $3 \mathrm{~s}$, covering the disturbance event.

However, the abrupt change detection-based automatic segmentation technique has been developed in a generic manner. So the aforesaid assumptions can be extended to other systems as well. For example, in the previous section, we discussed the segmentation of the power oscillation signals from a $60-\mathrm{Hz}$ frequency system using the same techniques. The sampling frequency can also be varied depending on the types of DFRs being used, which would usually result in more or less data samples to be available for analysis. The more data samples, the better the segmentation; however, the segmentation technique is robust enough to comply with fewer data samples. Segmentation does not depend on the signal duration, but rather on the abrupt changes in the signal model parameters, so any kind of signal can be segmented. The nonparametric approach using the WT is fast and accurate. The segmentation techniques can be applied to other application areas in power systems as well as completely different domains as future endeavors. These are mentioned below.

- The segmentation techniques can be applied for online signal segmentation as the first step toward online disturbance recognition and analysis [12].

- The abrupt change detection-based segmentation techniques can be successfully applied to other domains, such as segmentation of music signals for automatic music cognition [12].

- Possible applications involving the segmentation task, for example, in the dynamical system identification problems, seismology, financial indicators and analysis, failure detection, and isolation systems (FDI) [13], etc., could be considered further. 


\section{CONCLUSION}

Abrupt change detection using the WT and threshold method is quite effective in segmenting the signals originated by power system disturbances into event-specific sections. A novel synchronization algorithm for the different simultaneous disturbance recordings, segmented using abrupt change detection, is discussed in this paper. Using the synchronized and segmented analog signals and their respective matched binaries, further effective analysis can be performed for monitoring the performances of the protective relays (e.g., fastest relay operating time and autoreclosing of the CBs). The analysis of certain kinds of disturbances directly from the segmented recordings is also discussed in this paper. The methods, algorithms, and applications presented in this paper facilitate fast signal segmentation, relevant disturbance analysis, and reliable relay performance monitoring along with effective disturbance signal processing for further automatic disturbance recognition and analysis. The generic segmentation techniques could be used in other application areas in power systems and other domains as well.

\section{ACKNOWLEDGMENT}

The authors would like to thank P. Keller and Eskom Transmission, South Africa, for providing all real disturbance signal recordings.

\section{REFERENCES}

[1] P. F. Craigmile and D. B. Percival, Wavelet-based trend detection and estimation, Dept. Statist., Appl. Phys. Lab., Univ. Washington. Seattle, WA, 2000.

[2] D. L. Donoho and I. M. Johnstone, "Ideal spatial adaptation by wavelet shrinkage," Biometrika, vol. 81, no. 3, pp. 425-455, 1994.

[3] M. Chantler, P. Pogliano, A. Aldea, G. Tornielli, T. Wyatt, and A. Jolley, "The use of fault-recorder data for diagnosing timing and other related faults in electricity transmission networks," IEEE Trans. Power Syst., vol. 15, no. 4, pp. 1388-1393, Nov. 2000.

[4] E. Stokes-Waller, "Automated digital fault recording analysis on the eskom transmission system," presented at the Southern African Conf. Power System Protection, South Africa, 1998, unpublished.
[5] IEEE Standard Common Format for Transient Data Exchange (COMTRADE) for Power Systems, IEEE Std. C37.111-1991, Feb. 1991, Version 1.8 .

[6] A. Ukil and R. Živanović, "Detection of abrupt changes in power system fault analysis: A comparative study," in Proc. Southern African Universities Power Engineering Conf., Johannesburg, South Africa, Jan. 2005.

[7] A. Ukil and R. Živanović, "Abrupt change detection in power system fault analysis using adaptive whitening filter and wavelet transform," Elect. Power Syst. Res., vol. 76, no. 9-10, pp. 815-823, Jun. 2006.

[8] S. Mallat, A Wavelet Tour of Signal Processing. New York: Academic, 1998.

[9] I. Daubechies, Ten Lectures on Wavelets. Philadelphia, PA: Society for Industrial and Applied Mathematics, 1992.

[10] P. Keller, Analysis of digital records South Africa, Tech Rep., Apr. 2004, Eskom Transmission.

[11] D. R. Vega, A. R. Messina, and G. E. Harper, "Analysis of inter-area oscillations via non-linear time series analysis techniques," presented at the Power Syst. Comput. Conf., Liege, Belgium, Aug. 2005.

[12] A. Ukil, "Abrupt change detection in automatic disturbance recognition in electrical power systems," Ph.D. dissertation, Dept. Math. Tech., Tshwane Univ. Technol., Pretoria, South Africa, 2005.

[13] M. Basseville and I. V. Nikoforov, Detection of Abrupt Changes-Theory and Applications. Englewood Cliffs, NJ: Prentice-Hall, 1993.

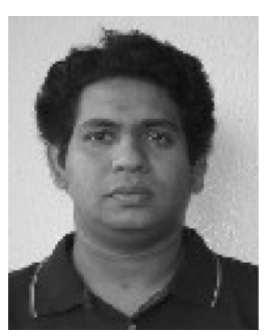

Abhisek Ukil (S'06) received the B.E. degree in electrical engineering from the Jadavpur University, Calcutta, India, in 2000 and the M.Sc. degree in electronic systems and engineering management from the University of Applied Sciences, South Westphalia, Soest, Germany, and Bolton Institute, Bolton, U.K., in 2004. He received the Dr. Tech degree from the Department of Mathematical Technology, Tshwane University of Technology, Pretoria, South Africa, in 2006.

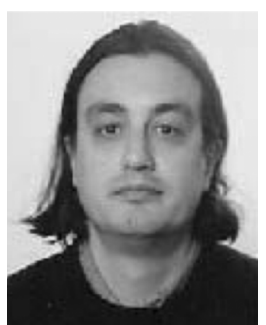

Rastko Živanović (M'97) received the Dipl. Ing. and M.Sc. degrees from the University of Belgrade, Belgrade, Serbia, in 1987 and 1991, respectively, and the Ph.D. degree from the University of Cape Town, Cape Town, South Africa, in 1997.

Currently, he is Professor with the Faculty of Engineering at Tshwane University of Technology, Pretoria, South Africa, where he has also been Lecturer and Senior Lecturer. His research interests include power system protection and control. 